Dermatologica, 1990;181:339

\title{
Retinoids 10 Years On
}

An International Symposium

Organized by the Department of Dermatology University of Geneva (Switzerland) November 16-17, 1990

Abstracts

of Invited Lectures and Free Communications

KARGER 